\title{
Tunable coaxial resonators based on silicon optical fibers
}

\author{
F. H. Suhailin, ${ }^{1,2}$ N. Healy, ${ }^{1}$ M. Sumetsky, ${ }^{3}$ L. Xiao, ${ }^{1}$ J. Ballato, ${ }^{4}$ A. N. Dibbs, ${ }^{5}$ U. Gibson, ${ }^{5}$ and A. C. Peacock ${ }^{1}$ \\ ${ }^{1}$ Optoelectronics Research Centre, University of Southampton, SO17 1BJ, United Kingdom \\ ${ }^{2}$ Physics Department, Faculty of Science and Technology, University Malaysia Terengganu, 21300 Kuala Terengganu, Malaysia \\ ${ }^{3}$ Engineering and Applied Science, Aston University, B4 7ET, United Kingdom \\ ${ }^{4}$ COMSET, School of Materials Science and Engineering, Clemson University, SC 29634, USA \\ ${ }^{5}$ Department of Physics, Norwegian University of Science and Technology, 7491 Trondheim, Norway \\ acp@orc.soton.ac.uk
}

\begin{abstract}
Thermal tuning of a coaxial fiber resonator with a silica cladding surrounding an inner silicon core is investigated. By pumping the silicon with below bandgap light, it is possible to redshift the WGM resonances.

OCIS codes: (140.4780) Optical Resonator; (140.3948) Microcavity Devices; (160.6000) Semiconductor Materials.
\end{abstract}

Whispering gallery mode (WGM) microresonators with ultra-high quality factors $(Q)$ and small mode volumes hold great potential for the construction of compact, low power photonic devices. To date, some of the largest $Q$ factors $\left(10^{8}\right)$ have been obtained in silica fiber resonators owing to the extremely low material loss and ultra-smooth outer surfaces [1]. However, compared to crystalline materials, silica has limited functionality, i.e., lower thermal and Kerr nonlinearities, which restricts the tunability of the resonators. In this paper we investigate a novel coaxial resonator geometry that has a thin micrometer scale silica outer cladding and an inner core consisting of silicon. The resonator is still silica-based, i.e., the circulating mode is confined by the pristine air/silica interface, but the silicon core can be modulated to tune the resonances.

The silicon core fiber was fabricated by packing a silica capillary with silicon granules, before heating and drawing through an oxyacetylene flame [2]. The resulting fiber had an outer silica diameter of $\sim 34 \mu \mathrm{m}$ and an inner silicon core diameter of $\sim 21 \mu \mathrm{m}$ (see inset Fig. 1(a)). For comparison, a pure silica fiber resonator was also fabricated by tapering a single mode fiber (SMF) to have a similar diameter. Fig. 1(b) shows the transmission spectrum recorded for the silicon core resonator using a tunable C-band CW source (coupled via a $2 \mu \mathrm{m}$ tapered fiber as in Fig. 1(a)), revealing that a series of mode families are excited with a free spectral range of FSR 17 nm, and a maximum loaded $Q$ factor of $Q_{l} \sim 3.9 \times 10^{4}$ at $\lambda_{r} \sim 1546.83 \mathrm{~nm}$. The larger FSR and $Q$ factors measured for this fiber compared to the tapered SMF (FSR 14nm and $Q_{I} \sim 1.6 \times 10^{3}$ ) indicates that there is some interaction with the silicon core which modifies the mode confinement. To investigate thermal tuning, both resonators were pumped with a Ti:Sapphire laser (200 fs, $80 \mathrm{MHz})$ at $810 \mathrm{~nm}$ using a tapered lens fiber (Fig. 1(a)). As the average power of the pump is increased, the strong absorption of silicon at this wavelength heats the inner core resulting in a thermal red-shift, as shown in Fig. 1(c). The maximum shift of $0.42 \mathrm{~nm}$ is obtained for a power of $\sim 66 \mathrm{~mW}$ coupled into the tapered lens. In contrast, no shift was measured in the SMF taper. Different pumping configurations will be considered to reduce the power and increase the response speed for future applications in all-optical signal processing.

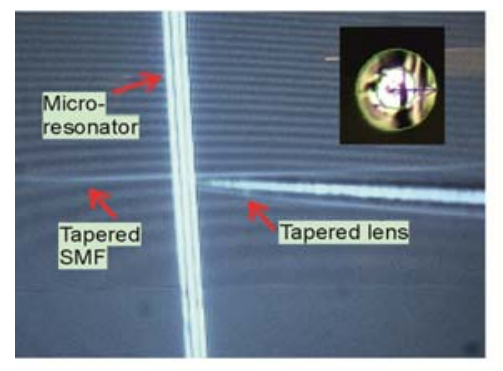

1(a)

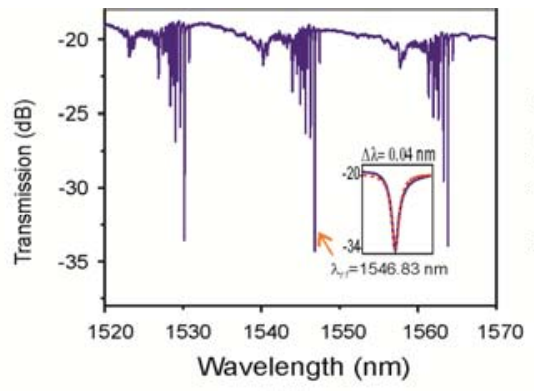

1 (b)

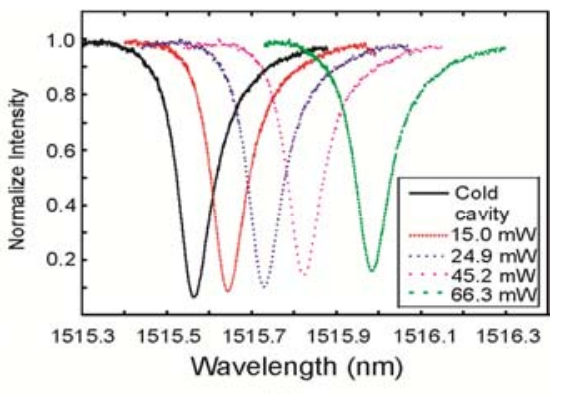

1 (c)

Fig. 1. (a) Image of the coaxial silicon fiber microresonator and tapered fiber couplers. Inset: Microscope image of the cross-section of the microresonator. (b) Transmission spectrum. Inset: Lorentian fit to determine $Q_{l}$. (c) Transmission as the function of side-pumped power.

\section{References}

[1] M. Pöllinger, D. O’Shea, F. Warken, and A. Rauschenbeutel, " Ultrahigh-Q Tunable Whispering-Gallery-Mode Microresonator," Phys. Rev. Lett. 103 , 053901, (2009).

[2] E. F. Nordstrand, A. N. Dibbs, A. J. Eraker, and U. J. Gibson, "Alkaline oxide interface modifiers for silicon fiber production," Opt. Mater. Express 3, 651, (2013). 